\title{
Comparative Analysis of VM Scheduling Algorithms in Cloud Environment
}

\author{
Puneet Himthani \\ M. E. Scholar \\ Department of CSE \\ TIEIT, Bhopal
}

\author{
Amit Saxena \\ Asso. Prof. \& H. O. D. \\ Department of CSE \\ TIEIT, Bhopal
}

\author{
Manish Manoria, PhD \\ Professor \\ Department of CSE \\ TIEIT, Bhopal
}

\begin{abstract}
Cloud computing is a pay per use model for providing convenient and on demand network access to sharable and configurable computing resources like networks, servers and applications that can be easily managed with minimal effort or service provider interaction. The various characteristics provided by cloud systems are Internet based computing, heterogeneity, on demand self service, pay per use model, location independent resource pooling, availability, scalability and ubiquitous network access.

Virtualization is a way of abstracting the physical resources to improve their utilization. It logically divides the resources between different users. A virtual machine is a logical implementation of a computer that operates in the similar manner as the computer system. A cloud computing environment requires a suitable algorithm for executing the various jobs provided to the system in a cost effective manner based on certain constraints. This task is performed by a VM scheduler using a suitable scheduling algorithm.
\end{abstract}

VM scheduling plays an important role in balancing the load of the system so that the utilization of the resources should be optimum. Better the scheduling policy, more the efficient operation of the cloud system.

\section{General Terms}

Cloud Computing, Virtualization, Virtual Machines, etc.

\section{Keywords}

Cloudlet Scheduling Algorithms, VM Scheduling Algorithms, Processor Scheduling Algorithms, etc.

\section{INTRODUCTION}

Cloud Computing is a modified form of distributed computing, parallel computing or grid computing. It is a convenient model for providing services and resources to its users through Internet.

It is highly reliable, scalable and efficient model for sharing resources and services between users. It is a pay per use model of computing means you have to pay only for what you have taken. Cloud models can be classified into a number of different categories. Commonly, there are two ways to categorize them, as:

\section{Service Models}

2. Deployment Models

1.1 Cloud Service Models: In this category, the cloud architectures are classified according to the services provided by them. It is also called as SPI model ${ }^{[14]}$. It can be further classified as:
1.1.1. Software as a Service (SaaS): The user can access the applications provided by the cloud service provider.

1.1.2. Platform as a Service (PaaS): The user can develop applications that can be provided to other users of the cloud environment and can deploy those applications on cloud through cloud service provider.

1.1.3. Infrastructure as a Service (IaaS): The user can get access over hardware resources provided by the cloud service provider and can use those resources for their own work. It includes services like storing the data on cloud.

1.2 Cloud Deployment Models: In this category, the cloud systems are classified on the basis of environment in which the users can access the services and resources deployed over the cloud. It can be categorized as:

1.2.1. Private Cloud: A cloud architecture that has been developed for specific organization or user. It is totally managed by the organization or user.

1.2.2. Community Cloud: A cloud architecture that has been developed in order to provide services to predefined number of organizations or a specific group of users. It is managed by a member of the participating organization or any specific user of the system.

1.2.3. Public Cloud: A cloud organization that has been developed to provide services and resources to any user that can become a member of that system. It can be managed by a third party organization that provides the cloud services or that has developed the cloud system.

1.2.4. Hybrid Cloud: A hybrid cloud is a combination of private cloud and public cloud.

\section{VM SCHEDULING}

Virtualization plays an important role in providing resources to the users efficiently in cloud environment. Virtualization can be done in various ways like server virtualization, memory virtualization, storage virtualization, etc. For efficiently achieving virtualization, virtual machines (VM) are designed.

$\mathrm{VM}$ is a logical instance of a computer system that can operate similarly to a system. The request of a user to access the physical resource in a cloud environment is first received by the VM and then VM assign that resource to the user based on the suitable policy or constraints specified. For this, VM schedulers are used. VM schedulers are used to dynamically 
assign the VM to users through which they can perform their specific operation in the cloud environment

By this, the utilization of the resources is improved as well as load balancing of all the systems is also managed. So, all the servers are equally sharing the load or services requested by the users. Each cloud environment incorporates a suitable VM scheduling policy for efficient utilization of resources ${ }^{[24]}$.

VM scheduling is necessary to maintaining the Quality of Service (QoS) and Service Level Agreements (SLA) specified by the cloud service provider to customers when the customer wants to take the cloud services.

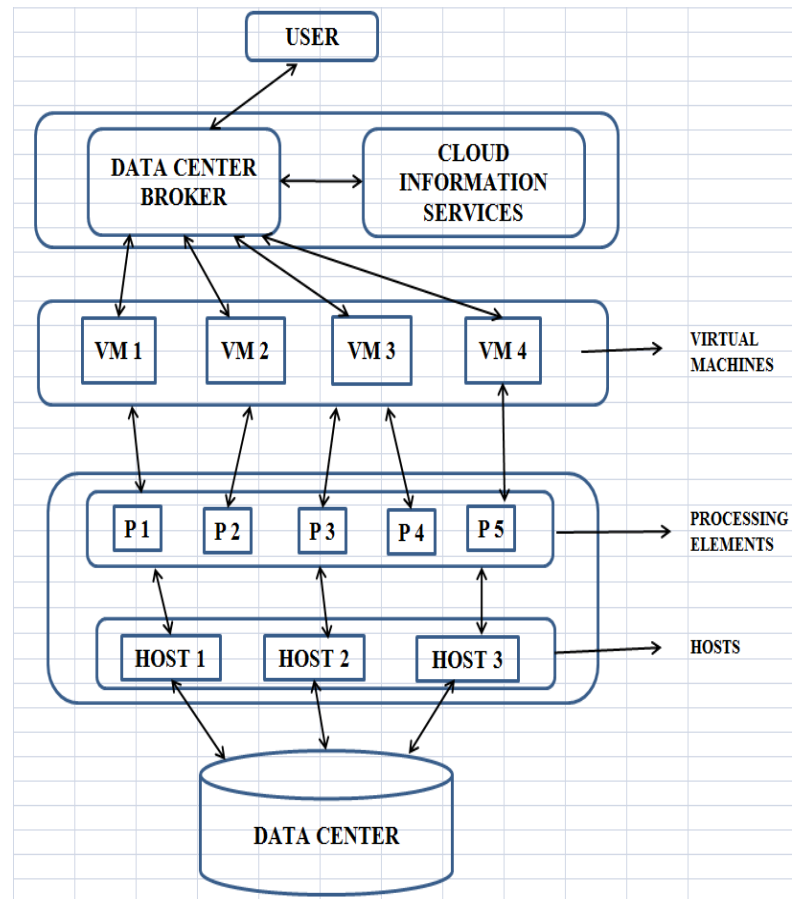

Fig.1 VM Scheduling in Cloud Computing Environment

VM Scheduling is a three step process ${ }^{[22]}$, as:

2.1. Resource Discovering and Filtering: Data Center Broker looks for the resources present in the system and take information about those resources.

2.2. Resource Selection: It is a deciding state in which the specific resource is selected based on certain constraints.

2.3. Task Submission: Task is provided to the selected resource for completion.

Virtualization techniques mainly aim at achieving scalability, availability, throughput and optimal resource utilization ${ }^{[14]}$. During operation, sometimes VM's are transferred from one system to another without disturbing the operation of other VM's operating in parallel. This is called VM migration. Migrations are performed in order to improve the utilization of the resources and to reduce the down time.

\section{SCHEDULING IN CLOUDSIM}

Cloudsim is a simulating tool that has been widely used for simulating cloud applications and related algorithms. It is an open source tool which is free to use. In cloudsim, scheduling has been performed at two different levels, viz. first between the Hosts and Virtual Machines for allocation of Processing
Elements (PE's) and second between the Virtual Machines and the Cloudlets, i.e., applications or processes to be executed over cloud. Commonly, the first is known as VM Scheduling and the second is known as Cloudlet Scheduling. Cloudsim basically supports two types of scheduling techniques at both levels, viz. Time Shared Scheduling and Space Shared Scheduling [27] [28]. Cloudsim also supports another type of VM Scheduling known as Time Shared over Subscription VM Scheduling ${ }^{[29]}$ and another type of Cloudlet Scheduling known as Dynamic Workload Cloudlet Scheduling.

In Space Shared Scheduling at any level, the sharing of Processing Elements between tasks or VM's is not possible. A PE can be allotted to new VM or a new task can be taken for execution only after completion of the previous task or successful execution of all tasks b a VM [30] [31]. While in Time Shared Scheduling, tasks can share the PE or the VM's can also share the PE's for execution.

Time Shared with Oversubscription VM Scheduling is basically used to improve the utilization of the cloud resources to a higher extent like improving the utilization of memory, PE's, etc. Dynamic Workload Cloudlet Scheduling is commonly employed in cases when there is only one cloudlet for execution that can be used to execute as an online web service.

Let us take two VM's VM1 and VM2 that will execute 8 tasks $\mathrm{t} 1, \mathrm{t} 2, \mathrm{t} 3, \mathrm{t} 4, \mathrm{t} 5, \mathrm{t} 6, \mathrm{t} 7$ and $\mathrm{t} 8$. Suppose tasks $\mathrm{t} 1, \mathrm{t} 2, \mathrm{t} 3$ and $\mathrm{t} 4$ are going to be executed by VM VM1 and tasks $t 5, \mathrm{t} 6, \mathrm{t} 7$ and t8 will be executed by VM VM2. Then the various possibilities for their execution will be:

1. Both VM's and Tasks will be Space Shared

2. VM's will be Space Shared and Tasks will be Time Shared

3. VM's will be Time Shared and Tasks will be Space Shared

4. Both VM's and Tasks will be Time Shared

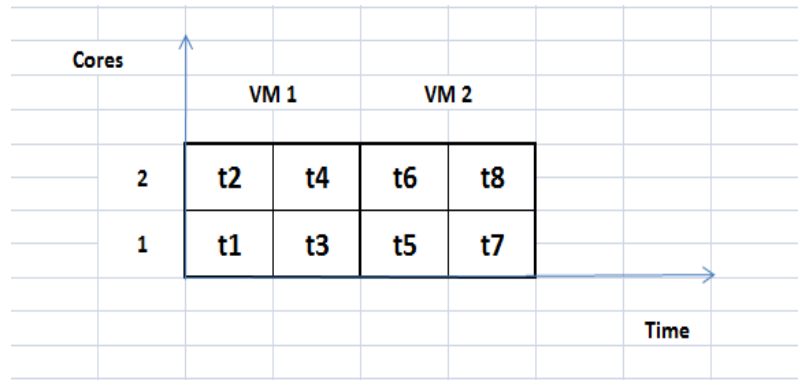

Fig. 2 Tasks and VM's both are Space Shared

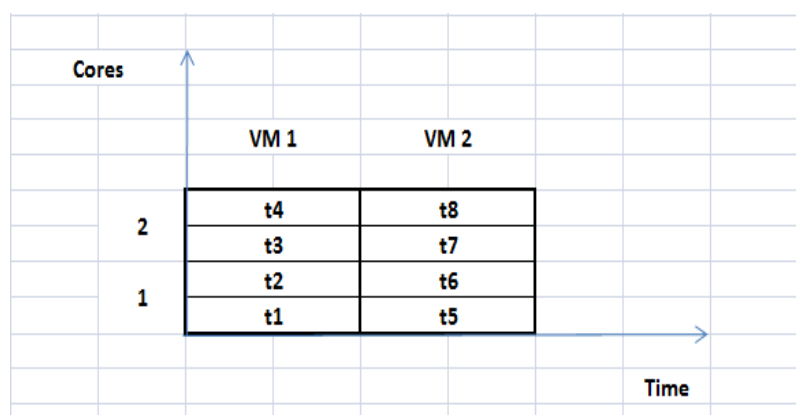

Fig. 3 Tasks are Time Shared \& VM's are Space Shared 


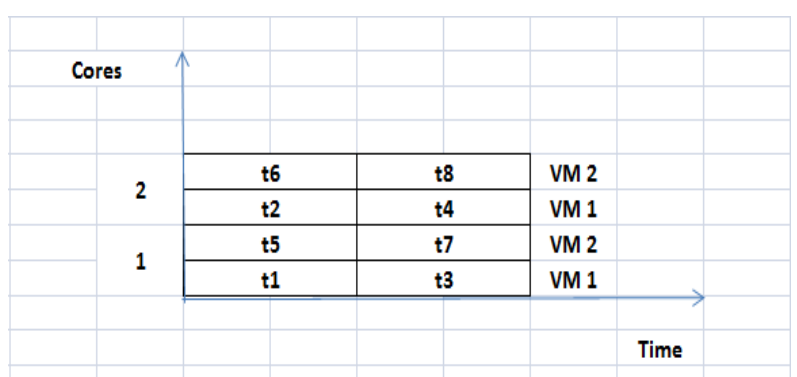

Fig. 4 Tasks are Space Shared \& VM's are Time Shared

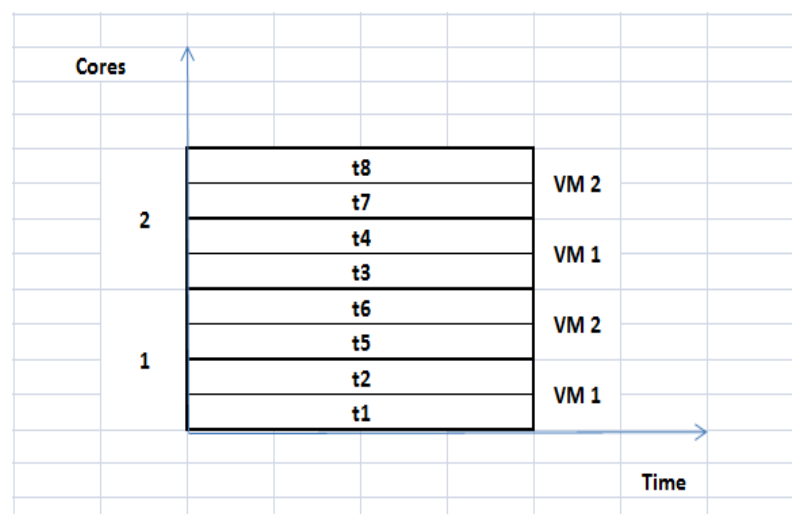

Fig. 5 Tasks \& VM's both are Time Shared

\section{RELATED WORK}

\subsection{Cloudlet Scheduling}

Ahn, Kim, Han, Choi and Huh ${ }^{[1]}$ proposed Memory Aware Cloud Scheduling based on the Cache behavior of the VM nodes. If the cache utilization of the nodes executing VM's is too high, then only migration will be performed as if not, it may lead to reduction in hit ratio or number of miss increases. Their technique is based on the concepts of various hardware architectures of distributed systems or multi computers like NUMA, COMA, etc. The results generated by them shows that the throughput and resource utilization of the system can be improved significantly up to $15-20 \%$.

Liu, Chen, Qiu, Cai and Huang ${ }^{[5]}$ proposed a modified scheduling algorithm based on FCFS scheduling. They proposed two level scheduling, viz. based on foreground VM's and background VM's. Processes in the Foreground VM's are scheduled on the basis of FCFS scheduling while processes in the Background VM's are scheduled on the basis of SJF scheduling approach. All background VM's are communicating with one or more foreground VM's. Any background VM whose current allocation of process is less than $96 \%$ can accommodate a new process otherwise it will not be allowed to accommodate a new process. Migrations are only performed during scheduling if certain processors remain idle for long periods of time. The results show that the response time can be significantly reduced by their algorithm.

Yeboah, Odabi and Abilimi ${ }^{[11]}$ proposed a modified round robin scheduling based on divisible load balancing theory. The processors are organized in the form of master - slave relationships with a master processor having a number of slave processors working under it. Then each job that has to be performed is sub divided into smaller jobs by the master processor. Then based on these sub jobs, VM's are initialized and the VM scheduler assigns these sub jobs to the specific
VM's based on their requirements. Then VM's execute the jobs assigned and after execution, dispatch the jobs and takes new job for execution, till all the jobs does not executed completely. Their results show that there has been significant improvement in the response time and the data center processing time of the cloud system.

Deore and Patil ${ }^{[12]}$ proposed energy efficient scheduling and allocation scheme based on the load provided to the system, i.e., during no workload, during minimum workload and during maximum workload. If there is no workload, then do not initialize any VM. During minimum workloads, then initialize VM's as per requests. During maximum workloads, perform migrations to improve the utilization of resources. The results show that the waiting time has been significantly reduced by applying their technique for cloudlet scheduling.

Xing, Liu and Ling ${ }^{[13]}$ proposed task scheduling algorithm based on neural networks and fuzzy logic. They had proposed a two level scheduling technique in which at first level, there is a QoS monitor that will collect and evaluate the process of task execution and at lower level, there are modules that will perform task scheduling. Tasks having similar requirements are combined together to form a task set. The task manager employs the fuzzy logic to perform its operation. Their results show that the throughput of the system has been improved to a certain extent.

James and Verma ${ }^{[21]}$ proposed weighted active monitoring load balancing algorithm to improve response time and execution time. In this approach, each VM has a different processing power capability and based on this capability, VM's are accommodated tasks or requests. If more than one VM are available, VM's are assigned requests in decreasing order of their capability and then to lower capable VM's according to the needs of the request, thus optimizing the resource utilization. Their results show that there has been significant improvement in the response time and the data center processing time of the cloud system.

Choudhary and Peddoju ${ }^{[22]}$ proposed an optimum scheduling algorithm. In this approach, first incoming tasks are grouped together based on their requirements and deadline constraints. Then these groups are prioritized according to their deadlines and profits. So, smaller tasks and more profitable tasks are scheduled first. Then the VM in which the turn-around time will be minimum is assigned the task group for execution. Also, VM will be selected on the basis of processing power and cost, so that the scheduling should be energy efficient. Their results show that the completion time or turnaround time of the cloudlets has been significantly improved and the cost of the data center infrastructure has been reduced.

$\mathrm{Wu}$, Liu, Ni, Yuan and Yang ${ }^{[26]}$ proposed market oriented hierarchical oriented strategy. It is a two level approach, viz. service level scheduling performed by the global scheduler and task level scheduling performed by the local scheduler. Global scheduler search for the available services and resources provided by the system. Local scheduler performs the task of VM allocation for optimal utilization of resources. Their results show that the completion time for cloudlet execution has been improved and the cost of the data center has been reduced.

\subsection{VM Scheduling / PE Scheduling}

Dodiya and Champaneria ${ }^{[32]}$ proposed Host selection technique for data intensive applications in cloud computing. Their technique is based on the communication delay between the host and the broker. The lower the delay, the more is the 
possibility to select that host for performing tasks. The results show that the completion time has been reduced to certain extent by their proposed approach.

Panchal and Kapoor ${ }^{[34]}$ proposed dynamic VM allocation algorithm based on clustering. Their results show that the utilization of the resources has been improved and the data center debts are also reduced. Their results also show that the algorithm performs better load balancing.

Sonam Rathore ${ }^{[35]}$ proposed a simple and efficient VM allocation policy based on first come first serve basis. Their results show that the resource utilization has been improved by their approach.

\section{COMPARATIVE ANALYSIS}

A number of algorithms exist for scheduling at both levels in cloud computing environment. To identify that which of them is most suitable depends upon a number of parameters like response time, turn-around time, cost, profit, energy consumption, security parameters, memory requirements, throughput, etc. The higher the trust between the user and the service provider, the better is the utilization of the resources and the scheduling approach.

Table 1: Comparison of various Cloudlet Scheduling Techniques in Cloud Environment based on following Parameters

\begin{tabular}{|c|l|c|c|c|c|c|}
\hline $\begin{array}{c}\text { S. } \\
\text { No. }\end{array}$ & \multicolumn{1}{|c|}{ Throughput } & $\begin{array}{c}\text { Response } \\
\text { Time }\end{array}$ & $\begin{array}{c}\text { Migration } \\
\text { Time }\end{array}$ & $\begin{array}{c}\text { Resource } \\
\text { Utilization }\end{array}$ & $\begin{array}{c}\text { Scalability \& } \\
\text { Reliability }\end{array}$ \\
\hline 1 & FCFS (Space Shared) ${ }^{[38]}$ & - & - & - & - & $\checkmark$ \\
\hline 2 & SJF $^{[39]}$ & $\checkmark$ & $\checkmark$ & - & $\checkmark$ & $\checkmark$ \\
\hline 3 & Round Robin (Time Shared) $^{[14]}$ & $\checkmark$ & $\checkmark$ & - & $\checkmark$ & $\checkmark$ \\
\hline 4 & Priority $^{[14]}$ & $\checkmark$ & $\checkmark$ & - & $\checkmark$ & $\checkmark$ \\
\hline 5 & Gang Scheduling $^{[14]}$ & $\checkmark$ & $\checkmark$ & - & $\checkmark$ & $\checkmark$ \\
\hline 6 & Memory Aware $^{[1]}$ & $\checkmark$ & - & $\checkmark$ & $\checkmark$ & $\checkmark$ \\
\hline 7 & Modified FCFS $^{[5]}$ & $\checkmark$ & $\checkmark$ & $\checkmark$ & $\checkmark$ & $\checkmark$ \\
\hline 8 & Modified RR $^{[11]}$ & $\checkmark$ & $\checkmark$ & - & $\checkmark$ & $\checkmark$ \\
\hline 9 & Energy Efficient $^{[12]}$ & $\checkmark$ & - & - & $\checkmark$ & $\checkmark$ \\
\hline 10 & Neuro Fuzzy $^{[13]}$ & $\checkmark$ & - & - & $\checkmark$ & - \\
\hline 11 & Weighted Active Monitoring $^{[21]}$ & $\checkmark$ & $\checkmark$ & - & $\checkmark$ & $\checkmark$ \\
\hline 12 & Optimal Scheduling $^{[22]}$ & $\checkmark$ & $\checkmark$ & - & $\checkmark$ & $\checkmark$ \\
\hline 13 & Market Oriented $^{[26]}$ & $\checkmark$ & $\checkmark$ & - & $\checkmark$ & $\checkmark$ \\
\hline 14 & Match Making $^{[17]}$ & $\checkmark$ & - & - & $\checkmark$ & $\checkmark$ \\
\hline 15 & Genetic Algorithm $^{[14]}$ & $\checkmark$ & - & $\checkmark$ & - \\
\hline
\end{tabular}

As far as VM scheduling / PE scheduling is concerned, it has been observed that the space shared scheduling is superior than time shared scheduling. This is because in time shared scheduling, some time has been lapsed in switching between tasks or switching between individual VM's for each processing element. But if we can incorporate the concept of Oversubscription in Time Shared Scheduling, we can improve the performance of Time Shared Scheduling to certain extent. Currently results show that time shared scheduling has a performance degradation of about $10 \%$ due to continuous switching between entities.

\section{CONCLUSION}

In this paper, we have provided an introduction to the need of scheduling in cloud computing environment. We have provided an introduction to various traditional cloudlet scheduling and VM scheduling algorithms and a number of already proposed algorithms by other researchers and professionals. We had provided a comparative analysis of these algorithms based on the certain parameters like throughput, response time, waiting time, scalability, etc. If we talk about energy efficient VM allocation, then by migrations and consolidations, we can improve the performance of the cloud environment and can reduce the power consumption of the resources. Here, we can switch a VM from one host to another, if the new host has capacity to execute that VM. Then first host will be shutted down till time, a new request does not arises in the system.

As already discussed, a number of algorithms have been proposed for this problem, still there are certain possibilities by which a more efficient algorithm can be designed. The new algorithm should be optimum, requires less memory, consume less power and should have quick response time, lower waiting time, lower slowdown time and lower turn-around time, yet it should be reliable, scalable and trustworthy. Also, the algorithm should perform as less number of migrations as possible for execution of all requests. So, all these issues are to be taken into consideration for implementing new cloudlet scheduling algorithm. In case of VM - PE scheduling, it should be taken care that there should not be starvation of any resource. 


\section{REFERENCES}

[1] Ahn, Kim, Han, Choi \& Huh "Dynamic Virtual Machine Scheduling in Clouds for Architectural Shared Resources".

[2] Ghribi, Hadji \& Zeghlache "Energy Efficient VM Scheduling for Cloud Data Centers: Exact Allocation and Migration Algorithms".

[3] Xu, Zhao, Hu \& Hu "Job Scheduling Algorithm based on Berger Model in Cloud Environment", Elsevier Journal of Advances in Engineering Software, 419 - 425, 45, April 2011 [0965 - 9978].

[4] Kong, Lin, Jiang, Yan \& Chu "Efficient Dynamic Task Scheduling in Virtualized Data Centers with Fuzzy Predictions", Elsevier Journal of Network \& Computer Applications, June 2010 [1084 - 8045].

[5] Liu, Chen, Qiu, Cai \& Huang "Scheduling Parallel Jobs using Migration \& Consolidation in the Cloud", Hindwai Publications of Mathematical Problems in Engineering, July 2012.

[6] Du, He \& Meng "Energy Efficient Scheduling of Tasks with Deadline in Virtualized Environments", Hindwai Publications of Mathematical Problems in Engineering, Spetember 2014

[7] Wei, Fan, Lu \& Ding "Application Scheduling in Mobile Cloud Computing with Load Balancing", Hindwai Journal of Applied Mathematics, September 2012.

[8] Xiao, Song \& Chen "Dynamic Resources Allocation using Virtual Machines for Cloud Computing Environment", IEEE Transactions on Parallel \& Distributed Systems, No. 6, Vol. 24, 2013.

[9] More, Kulkarni, Kolbe, Kothari \& Yahide "SLA Driven Load Balancing for Web Applications in Cloud Computing Environment", IISTE Journal of Information \& Knowledge Management, No. 1, Vol. 1, 2011 [2224 5758].

[10] Mazandarani \& Mormeni "QoS Aware Scientific Application Scheduling Algorithm in Cloud Environment", IISTE Journal of Computer Engineering \& Intelligent Systems, No. 12, Vol. 4, 2013 [2222 1719].

[11] Yeboah, Adabi \& Abilimi "Utilizing Divisible Load Sharing Theorem in Round Robin Algorithm for Load Balancing in Cloud Environment", IISTE Journal of Computer Engineering \& Intelligent Systems, No. 4, Vol. 6, 2015 [2222 - 1719].

[12] Deore \& Patil "Energy Efficient Job Scheduling \& Allocation Scheme for Virtual Machines in Private Clouds", International Journal of Applied Information Systems, No. 1, Vol. 5, January 2013 [2249 - 0868].

[13] Xing, Liu \& Ling "Neural Network PID Control Based Scheduling Mechanism for Cloud Computing", International Journal of Applied Mathematics \& Information Sciences, 789 - 796, No. 2, Vol. 9, March 2015 .

[14] Khan, Kapgate \& Prasad "A Review on Virtual Machine Management Techniques \& Scheduling in Cloud Computing", International Journal of Advanced Research in Computer Science \& Software Engineering, 838 845, Issue 12, Vol. 3, December 2013 [2277 - 128X].
[15] Shakya \& Karaulia "A Survey on Virtual Machine Scheduling in Cloud Environment", International Journal of Advanced Research in Computer Science \& Software Engineering, 727 - 729, Issue 2, Vol. 4, February 2014 [2277-128X].

[16] Bala \& Chana "A Survey of various Workflow Scheduling Algorithms in Cloud Environment", $2^{\text {nd }}$ National Conference on Information \& Communication Technology, 2011, Published in International Journal of Computer Applications.

[17] Prajapati, Rawal, Karamta \& Potdar "Comparison of Virtual Machine Scheduling Algorithms in Cloud Computing", International Journal of Computer Applications, No. 15, Vol. 83, December 2013 [0975 8887].

[18] Deore, Patil \& Bhargava "Energy Efficient Scheduling Scheme for Virtual Machines in Cloud Computing", International Journal of Computer Applications, No. 10, Vol. 56, October 2012 [0975 - 8887]

[19] Ezugwu, Buhari \& Junaidu "Virtual Machine Allocation in Cloud Computing Environment", International Journal of Cloud Applications \& Computing, 47 - 60, Issue 2, Vol. 3, April - June 2013

[20] Abirami \& Ramanathan "Linear Scheduling Strategy for Resource Allocation in Cloud Environment", International Journal on Cloud Computing: Services \& Architecture, No. 1, Vol. 2, February 2012.

[21] James \& Verma "Efficient VM Load Balancing Algorithm for a Cloud Computing Environment", International Journal on Computer Science \& Engineering, 1658 - 1663, No. 9, Vol. 4, Spetember 2012 [0975 - 3397].

[22] Choudhary \& Peddoju "A Dynamic Optimization Algorithm for Task Scheduling in Cloud Environment", International Journal of Engineering Research \& Applications, 2564 - 2568, Issue 3, Vol. 2, May - June 2012 [2248 - 9622].

[23] Soni \& Pahadiya "An Energy Efficient Cloud Computing using VM Scheduling Algorithm”, International Journal of Research in Engineering, Technology \& Management, Issue 4, Vol. 2, July 2014 [2347 - 7539].

[24] $\mathrm{Gu}, \mathrm{Hu}$, Zhao \& Sun "A New Resource Scheduling Strategy Based on Genetic Algorithm in Cloud Computing Environment", Journal of Computers, Academy Publishers, 42 - 52, No. 1, Vol. 7, January 2012.

[25] Li, Peng \& Zhang "A Scheduling Algorithm for Private Clouds", Journal of Convergence Information Technology, No. 7, Vol. 6, July 2011.

[26] Wu, Liu, Ni, Yuan \& Yang "A Market Oriented Hierarchical Scheduling Strategy in Cloud Workflow Systems", Springer, March 2011.

[27] Buyya, Ranjan \& Calheiros "Modeling and Simulation of Scalable Cloud Computing Environments and the Cloudsim Toolkit: Challenges and Opportunities".

[28] Mondal, Choudhary \& Islam "Performance Analysis of VM Scheduling Algorithm of Cloudsim in Cloud Computing", International Journal of Electronics \& 
Communication Technology, 49 - 53, Issue 1, Vol. 6, March 2015 [2230 - 7109].

[29] Householder \& Green "Impacts of Multi Class Oversubscription on Revenues and Performance in the Cloud", International Journal of Cloud Computing, 15 30, No. 1, Vol. 2, March 2014 [2326 - 7550].

[30] Semwal \& Rawat "Analysis of Cloud Service Provisioning Policies using a GUI Simulator", International Journal of Engineering and Technical Research, 341 - 344, Issue - 4, Vol. 2, April 2014 [2321 - 0869].

[31]Himani \& Sidhu "Comparative Analysis of Scheduling Algorithms of Cloudsim in Cloud Computing", International Journal of Computer Applications, No. 16, Vol. 97, July 2014 [0975 - 8887].

[32] Dodiya \& Champaneria "Host Selection Technique for Data Intensive Application in Cloud Computing", International Journal of Computer Science \& Technology, 969 - 972, Issue - 2, Vol. 3, June 2012 [0976 - 8491].

[33] Tian, Zhao, Xu, Zhung \& Sun “A Toolkit for Modeling and Simulation of Real Time Virtual Machine Allocation in a Cloud Data Center", IEEE Transactions on Automated Science \& Engineering, 2013.
[34] Panchal \& Kapoor "Dynamic VM Allocation Algorithm using Clustering in Cloud Computing", International Journal of Advance Research in Computer Science \& Software Engineering, Issue - 9, Vol. 3, September 2013 [2277-128X].

[35] Sonam Rathore "Efficient Allocation of Virtual Machine in Cloud Computing Environment", International Journal of Computer Science \& Informatics, 59 - 62, Issue - 3, Vol. 2, 2012 [2231 - 5292].

[36] Vikash "Dynamic Creation and Placement of Virtual Machine using Cloudsim", International Journal of Emerging Technology and Advanced Engineering, 675 679, Issue -8, Vol. 4, August 2014 [2250 - 2459].

[37] Agarwal \& Jain "Efficient Optimal Algorithm for Task Scheduling in Cloud Computing Environment", International Journal of Computer Trends and Technology, 344 - 349, No. 7, Vol. 9, March 2014.

[38] Behzad, Fotohi \& Effatparvar "Queue based Job Scheduling Algorithm for Cloud Computing", International Research Journal of Applied and Basic Sciences, 3785 - 3790, No. 12, Vol. 4, 2013.

[39] Kaur \& Kinger "A Survey of Resource Scheduling Algorithms in Green Computing”, International Journal of Computer Science \& Information Technologies, 4886 - 4890, No. 4, Vol. 5, 2014 [0975 - 9646]. 